\title{
mTOR is a selective effector of the radiation therapy response in androgen receptor-positive prostate cancer
}

\author{
Matthew J Schiewer ${ }^{1,2}$, Robert Den ${ }^{4}$, David T Hoang ${ }^{1,2}$, Michael A Augello ${ }^{1,2}$, \\ Yaacov $R$ Lawrence ${ }^{4}$, Adam P Dicker ${ }^{4}$ and Karen E Knudsen ${ }^{1,2,3,4}$
}

${ }^{1}$ Kimmel Cancer Center, Departments of ${ }^{2}$ Cancer Biology, ${ }^{3}$ Urology and ${ }^{4}$ Radiation Oncology, Thomas Jefferson University, 233 South 10th Street, BLSB 1008A, Philadelphia, Pennsylvania 19107, USA

(Correspondence should be addressed to K E Knudsen at Kimmel Cancer Center, Thomas Jefferson University; Email: karen.knudsen@kimmelcancercenter.org)

\begin{abstract}
lonizing radiation (IR) is used frequently in the management of multiple tumor types, including both organ-confined and locally advanced prostate cancer (PCa). Enhancing tumor radiosensitivity could both reduce the amount of radiation required for definitive treatment and improve clinical outcome. Androgen suppression therapy improves clinical outcomes when combined with radiation therapy but is associated with significant acute and chronic toxicities; hence, there is a clear need for alternative means to increase the therapeutic window of radiotherapy. Herein, it is demonstrated that the mammalian target of rapamycin (mTOR) inhibitors rapamycin (sirolimus) and temsirolimus limit both hormone therapy (HT)-sensitive and castration-resistant PCa (CRPC) cell proliferation as single agents and have a profound radiosensitization effect when used in combination with IR. Importantly, the observed radiosensitization was influenced by the treatment schedule, in which adjuvant administration of mTOR inhibitors was most effective in limiting PCa cell population doubling. This schedule-dependent influence on in vitro treatment outcome was determined to be the result of relative effects on the cell cycle kinetics. Finally, adjuvant administration of either mTOR inhibitor tested after IR significantly decreased clonogenic cell survival of both HT-sensitive and CRPC cells compared with IR alone. Taken together, these data demonstrate that inhibition of mTOR confers a radiosensitization phenotype that is dependent on relative cell cycle kinetics and provide a foundation for clinical assessment.
\end{abstract}

Endocrine-Related Cancer (2012) 19 1-12

\section{Introduction}

Prostate cancer (PCa) is the most frequently diagnosed non-cutaneous malignancy and the second leading cause of death due to cancer in men in the United States (Jemal et al. 2010). Treatment options for localized disease include watchful waiting, surgery, and radiotherapy (RT; Klein et al. 2009). In the context of definitive treatment, adjuvant therapy after radical prostatectomy, and in some cases metastatic disease, RT is becoming of increasing significance for successful management of PCa (Kwok \& Yovino 2010).

Androgens and the cognate receptor (androgen receptor (AR)) have a well-described function in all stages of PCa.
If disseminated at the time of diagnosis, first-line therapy is targeted against the AR signaling axis. Suppression of AR activity is achieved by using GnRH agonists that induce ligand depletion (chemical castration) and is sometimes used in combination with direct AR antagonists (such as bicalutamide; Klotz 2006, Loblaw et al. 2007, Taplin 2007, Chen et al. 2008, Knudsen \& Scher 2009). For locally advanced or high-risk disease, RT is frequently used, thus underscoring the need to delineate the impact of combination therapy. AR-directed therapeutics is initially effective due to the dependence of this tumor type on AR signaling; however, after a median time of 2-3 years, tumors recur and are deemed 'castration resistant' 
(castration-resistant PCa (CRPC)). CRPC development is highly attributed to inappropriate resurgence of AR activity, which occurs despite the absence of circulating serum androgens and administration of direct AR antagonists (Knudsen \& Scher 2009, Yuan \& Balk 2009). Strikingly, few therapeutic options have shown efficacy against this stage of the disease, and a major goal of current translational research is to develop means for preventing or delaying progression to CRPC. One means by which PCa cells bypass AR-directed therapeutics involves upregulation of rapamycin (Rapa)sensitive signaling (Mousses et al. 2001), and that combining mammalian target of rapamycin (mTOR) inhibition with AR-directed therapies prolongs hormone sensitivity in xenograft models of PCa (Schayowitz et al. 2010). Moreover, AR is known to promote mTOR activity (Xu et al. 2006b), thus suggesting that combining mTOR- and AR-directed therapeutics may cooperate to improve cellular and clinical responses to therapy.

Given the poor outcomes associated with resurgent AR activity and CRPC development, it is imperative to develop new means for enhancing therapeutic efficacy and thus to prevent the transition to CRPC. In patients with locally advanced PCa treated with RT alone, the 5 -year disease-free survival rate is $40 \%$ (Bolla et al. 2002). Therefore, improving the overall efficacy of RT could be of significant clinical benefit. Several potential mechanisms lead to RT failure, including altered proliferative and pro-survival potential, both of which are frequently observed in PCa.

A frequent genetic lesion that leads to both events is loss of PTEN function. Sixty percent of PCa demonstrate loss of heterozygosity at the PTEN locus (Cairns et al. 1997, McMenamin et al. 1999). Decreased expression of PTEN has been detected in $85 \%$ of primary PCa tumors compared to normal prostatic tissue of the same patient (Kremer et al. 2006), and patients with tumors harboring mutant PTEN have decreased survival, higher metastatic frequency, and higher prostate-specific antigen (PSA) levels, suggesting higher AR activity (Pourmand et al. 2007); therefore, PTEN is one of the most frequently altered genes in human $\mathrm{PCa}$ and is associated with lethal tumor phenotypes. The PTEN phosphatase serves at the molecular level to counteract the functions of phosphoinositide 3-kinase, which promotes proliferation and cell survival, in part through activation of mTOR (Sansal \& Sellers 2004). Akt serves as an intermediate signaling molecule for mTOR, which is a serine/threonine kinase that mediates cell growth, proliferation, survival, protein translation, and other oncogenic functions.

mTOR activity is often deregulated in Pca (Kremer et al. 2006), in part due to the prevalence of PTEN dysfunction. Genomic deletion of PTEN is associated with both increased Akt activation and AR activity (Sircar et al. 2009). mTOR mediates proliferation in $\mathrm{PCa}$ cells, at least in part, due to androgen-induced upregulation of D-type cyclin translation (Gao et al. 2003, Xu et al. 2006b). This event is suggested to, therefore, promote cell cycle progression. In addition, mRNA translation events that are dependent on mTOR are rapidly activated in response to ionizing radiation (IR), resulting in DNA repair and survival (Braunstein et al. 2009). As such, the mTOR signaling pathway is a potential target for enhancing RT efficacy and improving therapeutic intervention in $\mathrm{PCa}$.

Pharmacological mTOR inhibition has been demonstrated to block the induction of the proliferative, prosurvival, and oncogenic functions of mTOR (Hidalgo \& Rowinsky 2000), with remarkable effects in PTENdeficient tumors. mTOR inhibitors (e.g. everolimus) have been approved by the FDA for treatment of renal cell carcinoma based on a successful phase III clinical trial (Motzer et al. 2008); thus, mTOR is an established therapeutic target and mTOR inhibitors appear to be reasonably well tolerated. At the cellular level, mTOR inhibitors have been shown to sensitize multiple tumor types to DNA damage-inducing agents, including IR, using both in vitro and in vivo models of human disease (Beuvink et al. 2005, Wu et al. 2005, Cao et al. 2006, Aissat et al. 2008, Morgan et al. 2008, Ekshyyan et al. 2009, Fung et al. 2009, Matsuzaki et al. 2009, Murphy et al. 2009, Saunders et al. 2010). Moreover, mTOR signaling has been implicated as a determinant of cell survival in response to DNA damage (Shen et al. 2007).

This study assessed the impact of mTOR inhibition in clinically relevant models of hormone therapy (HT)sensitive PCa and CRPC tumor cells both alone and in combination with RT. Survival analyses revealed that mTOR inhibitors sensitized both HT-sensitive PCa and CRPC cells to IR at clinically attainable doses. The impact of sequence of mTOR inhibition as a radiosensitizer was also assessed, where it was observed that the radiosensitization events were influenced by the scheduling. Strikingly, mTOR inhibitors were most effective at conferring radiosensitization effects when administered in the adjuvant setting. Schedule dependence was determined to be due to cell cycle kinetics, in which neoadjuvant use of mTOR inhibitors limited entry of the cells into a state of active DNA replication. On combining these studies, it is demonstrated that mTOR inhibitors radiosensitize AR-positive PCa cells dependent on treatment schedule and relative cell cycle inhibition and provide evidence of a viable combinatorial treatment strategy. 


\section{Materials and methods}

\section{Cell culture and reagents}

LNCaP, C4-2, and LAPC4 cells were cultured under standard conditions at $37{ }^{\circ} \mathrm{C}$ and $5 \% \mathrm{CO}_{2}$ as described previously (Sharma et al. 2010). Rapa was obtained from Calbiochem (San Diego, CA, USA) and dissolved in DMSO. Temsirolimus (Tem) was obtained from LC Laboratories (Woburn, MA, USA) and dissolved in ethanol.

\section{lonizing radiation}

A Panatek orthovoltage X-ray irradiator was used to deliver IR. The irradiator was calibrated daily using a Victoreen dosimeter.

\section{Cell counting/survival}

To monitor cell number over time, indicated cells were seeded on poly-L-lysine-coated dishes at equal densities and subjected to treatment/schedules described. At the time of harvest, cells were trypsinized and counted using Trypan Blue exclusion and a hemacytometer. Total cell number was determined from at least three independent experiments of three biological replicates.

\section{Cell cycle analysis/bivariate FACS}

To monitor bromodeoxyuridine (BrdU) incorporation/DNA content, LNCaP cells were seeded on polyL-lysine-coated dishes at equal densities and subjected to treatment/schedules described. Two hours prior to harvest, cells were incubated with BrdU (1:1000, Amersham Cell Proliferation Labeling Reagent, GE Healthcare, Buckinghamshire, UK). After labeling, cells were trypsinized and harvested, washed with PBS, and then re-suspended in PBS. Cells were then fixed with cold $100 \%$ ethanol, pelleted, then re-suspended in $2 \mathrm{M} \mathrm{HCl}$, and incubated for $20 \mathrm{~min}$ at ambient temperature. $\mathrm{HCl}$ was neutralized with $0.1 \mathrm{M}$ sodium tetraborate, washed with IFA buffer, followed by a wash with IFA buffer supplemented with $0.5 \%$ Tween 20 , then re-suspended in IFA buffer containing $6 \%$ FITC-conjugated anti-BrdU anti-sera (BD Biosciences, San Diego, CA, USA), and incubated for $45 \mathrm{~min}$. Cells were then washed with IFA buffer supplemented with $0.5 \%$ Tween 20, stained with propidium iodide $(0.2 \mathrm{~g} / \mathrm{ml})$, and subjected to flow cytometry. Samples were quantified on a Coulter Epics XL-MCL using XL System II Software (Beckman Coulter, Brea, CA, USA) and analyzed using FlowJo Software (Tree Star, Inc., Ashland, OR, USA). To monitor only the DNA content,
LNCaP cells were seeded on poly-L-lysine-coated dishes at equal densities and subjected to treatment/schedules described. Cells were trypsinized and harvested, washed with PBS, and then re-suspended in PBS. Cells were then fixed with cold $100 \%$ ethanol, pelleted, stained with propidium iodide $(0.2 \mathrm{~g} / \mathrm{ml})$, and subjected to flow cytometry. Samples were quantified on a Coulter Epics XL-MCL using XL System II Software (Beckman Coulter) and analyzed using FlowJo Software (Tree Star, Inc.).

\section{Clonogenic cell survival}

Exponentially growing cells were trypsinized and counted using Trypan Blue exclusion. Cells were diluted serially to appropriate concentrations and plated into $50 \mathrm{ml}$ tissue culture flasks in triplicate for $24 \mathrm{~h}$. Then, cells were treated with increasing doses of IR $(0$, 2, 4, 6, and $8 \mathrm{~Gy}$ ). After $24 \mathrm{~h}$, cells were treated with Rapa (10 nM), Tem (10 nM), or nothing. After 14 days of incubation, the colonies were fixed and stained with $4 \%$ formaldehyde in PBS containing $0.05 \%$ crystal violet. Colonies containing $>50$ cells were counted. Surviving fraction was calculated as (mean colony counts $) /($ cells inoculated $) \times($ plating efficiency $))$, in which plating efficiency was defined as (mean colony counts)/(cells inoculated for un-irradiated controls).

\section{Results}

\section{Single-agent mTOR inhibitors or IR limit HT-sensitive PCa cell growth}

mTOR activity has been observed to be increased in PCa through various mechanisms and upstream signaling defects. To challenge the consequence of mTOR inhibition in PCa, HT-sensitive cells were treated with increasing doses of two pharmacological inhibitors of mTOR activity, Rapa and Tem. It has been demonstrated that in this cell type, androgens induce mTOR signaling that culminates in cell cycle progression via an increased translation of cyclin D1 (Xu et al. 2006b), which is part of the molecular machinery responsible for the $\mathrm{G}_{1}-\mathrm{S}$ phase transition (Baldin et al. 1993). Consistent with previous reports, mTOR inhibition resulted in decreased cell number after $72 \mathrm{~h}$ of treatment (Fig. 1A; van der Poel et al. 2003). As demonstrated, there was no significant difference between either of the mTOR inhibitors tested with regard to response at any of the doses tested. As IR is used as definitive treatment for localized, HT-sensitive PCa, the effect of IR on HT-sensitive cells was assessed. These results demonstrate a dose-dependent decrease in population cell doubling after exposure to IR (Fig. 1B). 

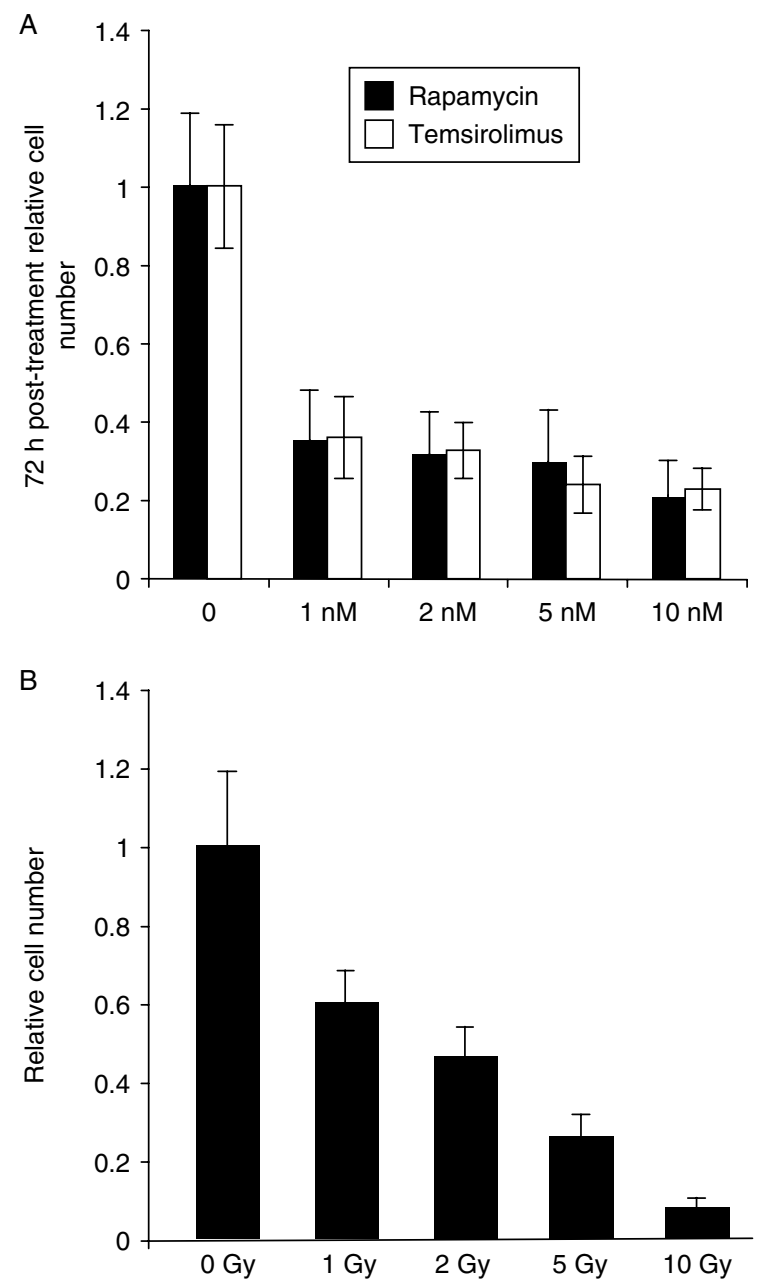

Figure 1 mTOR inhibitors and ionizing radiation (IR) are sufficient to limit hormone therapy-sensitive prostate cancer (PCa) cell doubling as single agents (A). LNCaP cells were treated with indicated doses of rapamycin, temsirolimus, or vehicle control. $72 \mathrm{~h}$ after treatment, cell number was assessed via Trypan Blue exclusion using a hemacytometer. Cell number in the vehicle controls was set to ' 1 '. Averages of three independent experiments and S.D. are shown. (B) LNCaP cells were exposed to the indicated doses of IR. $168 \mathrm{~h}$ after treatment, cell number was assessed via Trypan Blue exclusion using a hemacytometer. Cell number in un-irradiated controls was set to ' 1 '. Averages of three independent experiments and S.D. are shown.

Taken together, these data demonstrate that singleagent mTOR inhibitors and IR affect HT-sensitive cells. Additionally, there was no observable difference in the efficacy of Rapa and Tem in this context.

\section{Combining mTOR inhibition and IR is more effective than single agent in limiting HT-sensitive PCa cell number}

While IR is a frequently used treatment modality for locally advanced disease, there is a $10-60 \%$ recurrence rate (Allen et al. 2007), suggesting that means to improve the efficacy of RT is a significant clinical need. Based on this premise, and the observation that mTOR signaling is both involved in PCa cell cycle progression/survival (Gao et al. 2003, Xu et al. 2006b) and induced by IR (Tirado et al. 2003, Shen et al. 2007), the impact of mTOR inhibition on the response to IR was determined in HT-sensitive cells. To determine whether scheduling of the treatment affected outcome, a strategy was used to test concurrent (Schedule I), neo-adjuvant (Schedule II), and adjuvant (Schedule III) mTOR inhibitor administration. The time from final treatment to assessment of outcome was identical for all schedules tested. Cells were sensitized to IR when mTOR inhibition was co-administered (Fig. 2A; compare IR, Rapa, and Tem alone to Rapa + IR and Tem + IR). To assess impact on the neoadjuvant context, mTOR inhibitors were administered $48 \mathrm{~h}$ prior to IR treatment; as shown in Fig. $2 \mathrm{~B}$, there was a significant decrease in cell number following this treatment schedule (compare single agents vs combination). Finally, adjuvant mTOR inhibition conferred radiosensitization effects (Fig. 2C). Notably, Schedule III was most effective in limiting cell doubling $(\sim 15 \%$ of control) when compared with Schedule I ( $\sim 20 \%)$ or Schedule II $(\sim 23 \%)$, suggesting that scheduling of treatment should be considered in therapeutic design. The impact of schedule was likely attributed to relative effects on cell cycle progression and was conserved in another HT-sensitive PCa cell model (LAPC4; Supplementary Figure 1, see section on supplementary data given at the end of this article). The LAPC4 model maintains wild-type PTEN (Whang et al. 1998) and harbors a mutant p53 allele (van Bokhoven et al. 2003), suggesting that neither PTEN or p53 status alters the radiosensitization effect of mTOR inhibitors. Although the contribution of PTEN status to mTOR inhibitor sensitivity has been documented, data herein demonstrate that both PTEN-proficient and PTENdeficient cells can be radiosensitized by mTOR inhibition. Of note, the PTEN-proficient cell line LAPC4 may be intrinsically more radioresistant compared to the other model systems used. This is not without precedent, as it as been demonstrated that this cell line is relatively insensitive to chemotherapy (Xu et al. 2006a, Qian et al. 2010). However, mTOR inhibition still renders this cell type more sensitive to radiation. Taken together, these data demonstrate that combining mTOR inhibitors with IR is effective in limiting PCa cell number over time regardless of scheduling; however, adjuvant use of mTOR inhibitors may be most efficacious. 


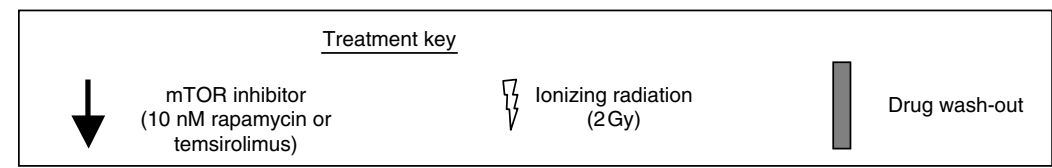

A

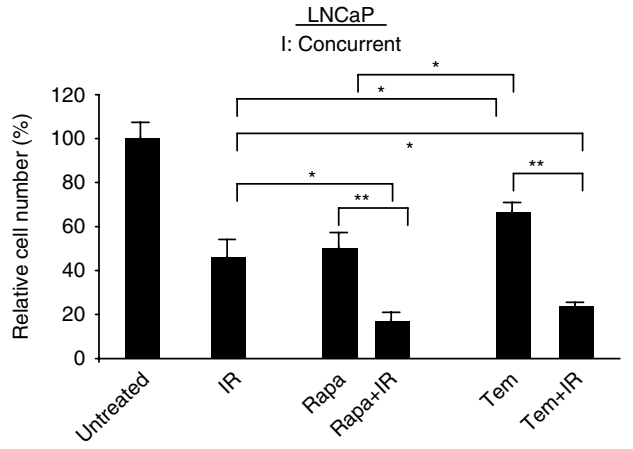

B

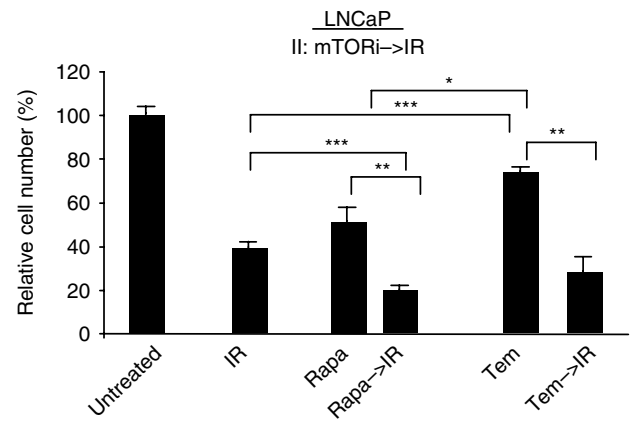

C
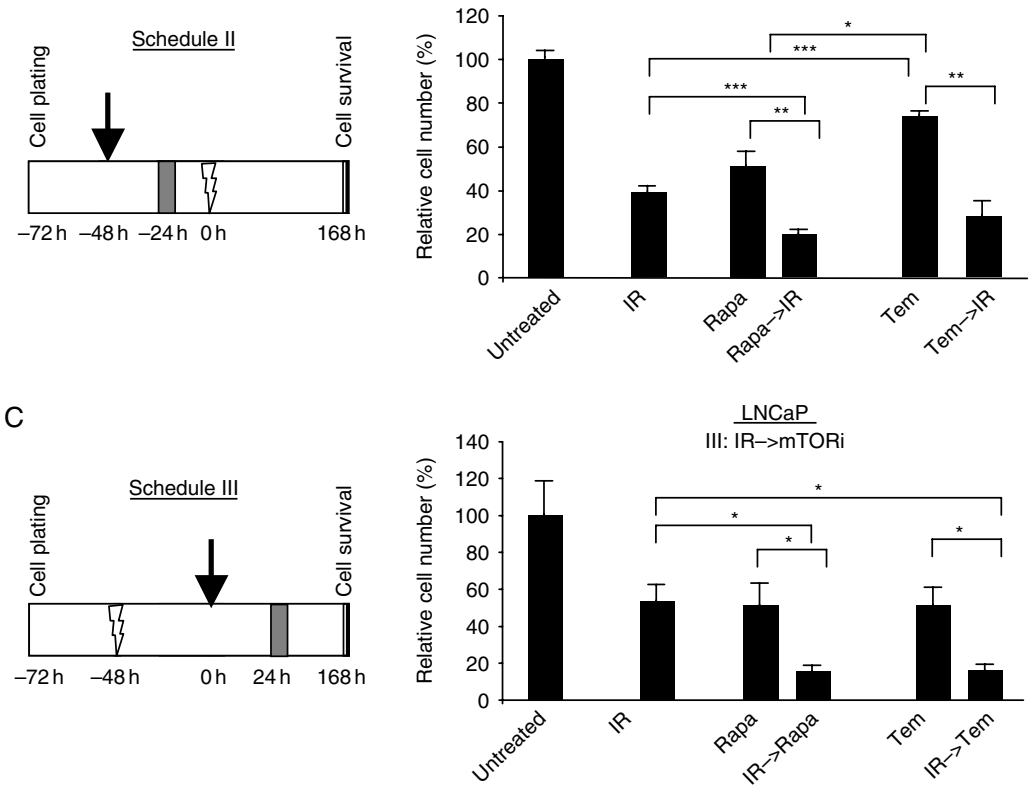

Figure 2 Schedule-specific radiosensitization of PCa cells by mTOR inhibition (A) Left panel: schematic of Schedule I treatment strategy (concurrent administration). As depicted, cells were seeded $72 \mathrm{~h}$ prior to final treatment, mTOR inhibitors, IR, or combination thereof were administered concurrently (Schedule I; set as time ' 0 '), drug was washed out $24 \mathrm{~h}$ later, and cell number was assessed $168 \mathrm{~h}$ after treatment. Right panel: LNCaP cells were treated with $10 \mathrm{nM}$ rapamycin (Rapa), $10 \mathrm{nM}$ temsirolimus (Tem), 2 Gy IR (IR), combination of rapamycin and IR (Rapa + IR), combination of temsirolimus and IR (Tem + IR), or vehicle control (untreated). Cell survival in the untreated control was set to 100\%; averages of three independent experiments and S.D. are shown. (B) Left panel: schematic of Schedule II (mTOR inhibitors as neoadjuvant). As depicted, cells were seeded $72 \mathrm{~h}$ prior to final treatment, administered $10 \mathrm{nM}$ of either mTOR inhibitor, which was washed out of culture media $24 \mathrm{~h}$ later, then $24 \mathrm{~h}$ after wash, which at time 0 was exposure to $2 \mathrm{~Gy} I \mathrm{R}$, and cell number was assessed $168 \mathrm{~h}$ post-IR. Right panel: same as in (A), but with neoadjuvant mTOR inhibitor administration. (C) Left panel: schematic of Schedule III (mTOR inhibitors as an adjuvant post-IR). Cells were seeded $72 \mathrm{~h}$ prior to final treatment, administered $2 \mathrm{~Gy} I \mathrm{R}$, and then treated $48 \mathrm{~h}$ prior to final treatment (time 0), in this case it was either $10 \mathrm{nM}$ rapamycin or temsirolimus. Right panel: same as in (A) and (B), but with adjuvant mTOR inhibitor administration. Statistical analysis of the indicated averages was performed using Student's $t$-test where ${ }^{\star} P<0.05,{ }^{\star \star} P<0.01$, and ${ }^{\star \star \star} P<0.001$. 


\section{mTOR inhibition radiosensitizes CRPC cells}

In addition to being used as a therapy for localized disease, IR is also used for local recurrence and metastases, when the cells have frequently become resistant to HT (CRPC cells). In the presence of androgens, mTOR inhibition sensitizes CRPC cells to IR (Fig. 3A). While there was less dependence on scheduling in this cellular context, Schedule III (adjuvant) remained the most effective in limiting cell doubling. To assess whether mTOR inhibition sensitizes CRPC cells to IR in a castrate environment, parallel studies were performed in steroid-depleted conditions. mTOR inhibitors retained the capacity to radiosensitize CRPC cells in the castrate condition (Fig. 3B), albeit to a lesser extent than observed in the presence of androgens

A
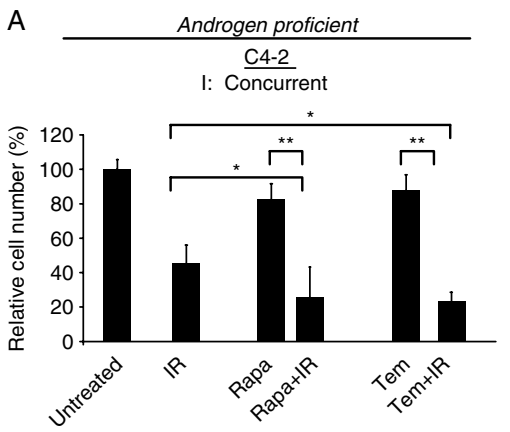

$\frac{\mathrm{C} 4-2}{\mathrm{TORi}->\mathrm{IR}}$

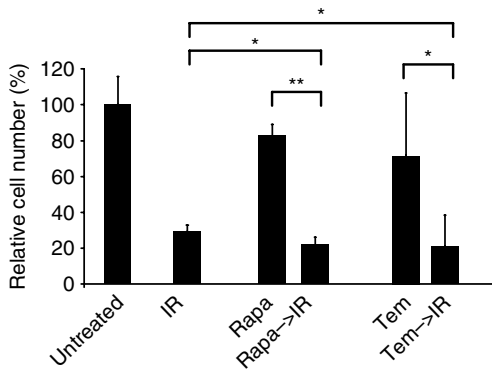

C4-2

III: IR->MTOR

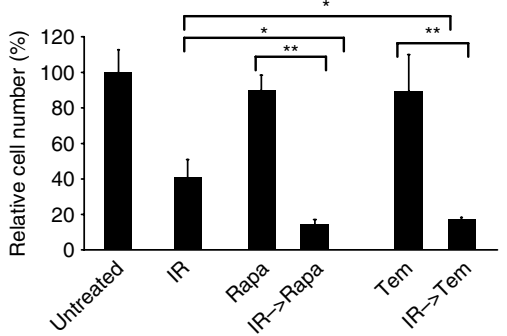

(compared to Fig. 3A). Regardless, Schedule III remained the most effective, which suggests that there is a potential cell cycle component involved in the efficacy of the combination treatment. Since mTOR inhibitors alone can suppress AR-dependent cyclin D1 accumulation and cell cycle progression, it was hypothesized that these cytostatic effects underlie the scheduling effects of mTOR inhibitors.

\section{Relative cell cycle inhibition in combination treatment is inversely correlated to efficacy of inhibiting population doubling}

To examine the relative cell cycle distribution of cells in each of the treatment schedules prior to irradiation, the amount of DNA in cell populations was determined
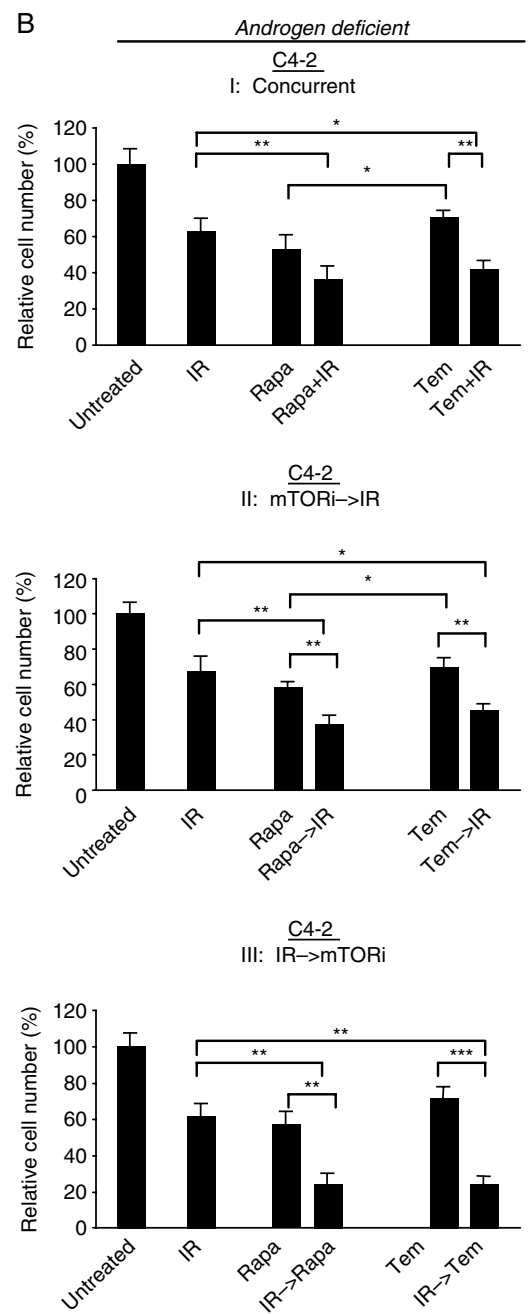

Figure 3 mTOR inhibitors sensitize CRPC cells to the effects of irradiation (A) C4-2 cells were treated as in Fig. 2. Top: mTOR inhibitor administration concurrent with IR (Schedule I). Middle: mTOR inhibitors administered as neoadjuvant to IR (Schedule II). Bottom: mTOR inhibitors administered as adjuvant to IR (Schedule III). (B) C4-2 cells were cultured in steroid-deprived media and treated as in (A). Cell survival in the untreated control is set to $100 \%$, averages of three independent experiments and s.D. are shown. Statistical analysis of the indicated averages was performed using Student's $t$-test where ${ }^{\star} P<0.05,{ }^{\star \star} P<0.01$, and ${ }^{\star \star \star} P<0.001$. 
by flow cytometry. There was a significant increase in $G_{1}$ enrichment in both the concurrent and the neoadjuvant schedules compared with the adjuvant, with a concomitant decrease in $\mathrm{G}_{2} / \mathrm{M}$ enrichment (Fig. 4A). This observed alteration in cell cycle distribution resulted in increased cells in a relatively radioresistant portion of the cell cycle $\left(\mathrm{G}_{1}\right.$; Yau et al. 1980) and a decrease in the number of cell in a relatively more radiosensitive portion of the cell cycle $\left(\mathrm{G}_{2} / \mathrm{M}\right.$; Sinclair \& Morton 1966) when mTOR inhibition was administered either concurrently or as a neoadjuvant. This same observation held true for CRPC cells as shown in Supplementary Figure 2A, see section on supplementary data given at the end of this article. In order to test the hypothesis that administration of mTOR inhibitors prior to the DNA-damaging insult of IR resulted in cytostatic effects that limited progression of cells to the radiosensitive cell cycle window, the relative change in S-phase progression was assessed for all treatment in the three schedules. There was significant inhibition of BrdU incorporation in both single-treatment mTOR inhibitor and IR in all schedules tested (Fig. 4A). The observed inhibition of S-phase progression was enhanced by combining mTOR inhibition and IR, but to a lesser extent in Schedule III (Fig. 4B). Representative PI/BrdU traces are provided in Supplementary Figure 2B, see section on supplementary data given at the end of this article. When these data were compared to the relative impact on cell number in Fig. 2 (Schedule III being the most effective in limiting population doubling), there was an apparent inverse correlation between relative cell cycle inhibition and inhibition of cell number. Therefore, while combinatorial treatment in Schedule III was least effective in limiting BrdU incorporation, it was this regimen that proved most effective in limiting cell number over time. To formally assess the impact of inhibition of cell cycle progression on relative treatment efficacy, cells were arrested with the DNA polymerase inhibitor aphidicolin (or not) prior to combination of mTOR inhibitors and IR (Schedule III). When the cell cycle was inhibited prior to administration of the Schedule III regimen, there was a significant alteration of the efficacy of combination
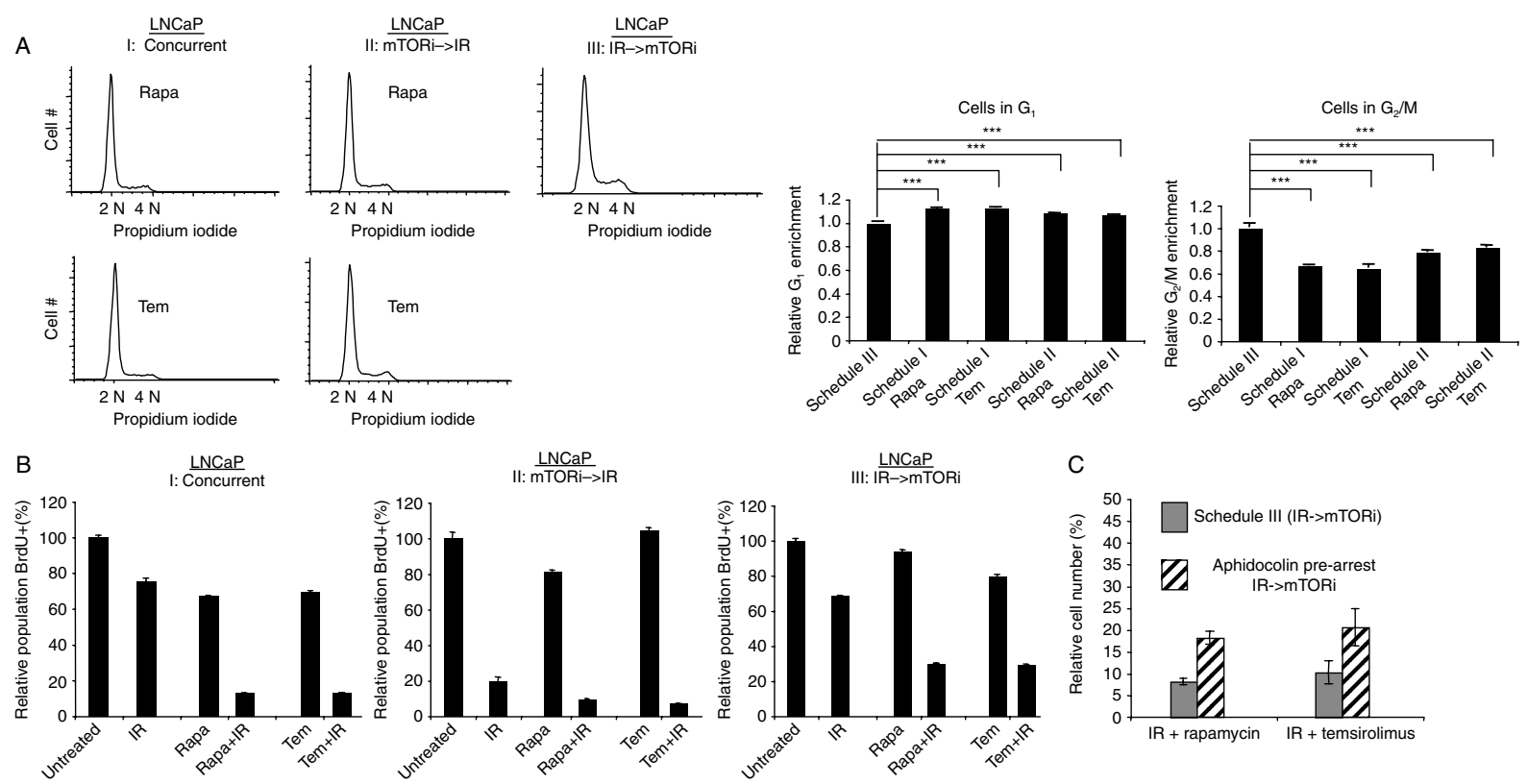

Figure 4 mTOR inhibitor-induced radiosensitization is a function of relative cell cycle inhibitory effect based on scheduling (A) Left panels: representative flow cytometry traces for each treatment Schedule that LNCaP cells were subjected to as depicted in Fig. 2 and harvested just prior to when IR would have been administered, fixed, and prepped for FACS analysis of DNA content as described in the Materials and methods section. Right panels: quantitation of the above. Data represented as relative $G_{1}$ (left) and $\mathrm{G}_{2} / \mathrm{M}$ (right) enrichment averages and S.D. of at least three independent experiments. Schedule III (adjuvant) is set to 1. (B) LNCaP cells were treated according to the schemata depicted in Fig. 2, then $24 \mathrm{~h}$ post treatment, cells were harvested, fixed, and prepped for FACS analysis of BrdU incorporation and DNA content as described in the Materials and methods section. The data shown depicts the averages and S.D. of at least three independent experiments analyzing the percent of the cell population that is BrdU positive compared to untreated control, which is set to $100 \%$. (C) LNCaP cells were either pre-arrested with vehicle control (gray bars) or aphidicolin (hashed bars) and then subjected to the mTOR inhibitors administered as an adjuvant to IR (Schedule III). Cell number was assessed $168 \mathrm{~h}$ after the last treatment by Trypan Blue exclusion and hemacytometer. Graph represents averages and S.D. of at least three independent experiments, with survival of untreated cells set to $100 \%$. ${ }^{\star \star \star} P<0.001$. 
treatment (Fig. 4C). Taken together, these data demonstrate that limiting cell cycle progression, either in the context of the scheduling or with another compound, prior to IR is less effective than using mTOR inhibitors in an adjuvant context. These data suggest that the anti-proliferative effect of mTOR inhibition prior to treatment is likely a detriment to therapeutic outcome, as the effects of IR may be greater in cells that are actively cycling, while the antisurvival effect of these compounds after IR may be of therapeutic benefit.

\section{mTOR inhibition combined with IR hinders clonogenic PCa cell survival}

To determine whether the observed mTOR inhibitormediated radiosensitization translated in long-term assays to significantly decrease in cell survival/clonogenicity, the Schedule III regimen was used in a clonogenic cell survival assay. Both Rapa and Tem when used in combination with IR significantly decrease clonogenic cell survival in HT-sensitive (Fig. 5A) and castration-resistant (Fig. 5B) cell models. These results, in a system that is a validated predictor of therapeutic response, indicate that adjuvant administration of mTOR inhibitors decreases $\mathrm{PCa}$ cell survival and replicative capacity.

\section{Discussion}

This study identifies mTOR inhibition as a therapeutic approach that, when combined with IR, suppresses cancer cell growth. While both IR and the two mTOR inhibitors tested (Rapa and Tem) showed single-agent efficacy in limiting PCa cell doubling at clinically relevant doses (Fig. 1), the data presented in this study provide evidence that when the combination of IR and mTOR inhibition is used, there is an additive effect in limiting both HT-sensitive PCa and CRPC cell doubling (Fig. 2). This cooperative effect was observed to be dependent on the scheduling of the treatment in that treatment of PCa cells with mTOR inhibitor(s) after IR treatment (adjuvant, Schedule III) resulted in the most additive effect as determined by both cell number and BrdU incorporation (Figs 2 and 4). Further, this observation was supported by the finding that arresting the cell cycle prior to administration of the most effective schedule reduced the efficacy of this treatment regimen (Fig. 4B). Finally, using clonogenic cell survival modeling, which is a predictor of in vivo efficacy (Wilson et al. 1984, Hirabayashi et al. 1987, Yung 1989), it was observed that adjuvant administration of either of the mTOR inhibitors tested resulted
A
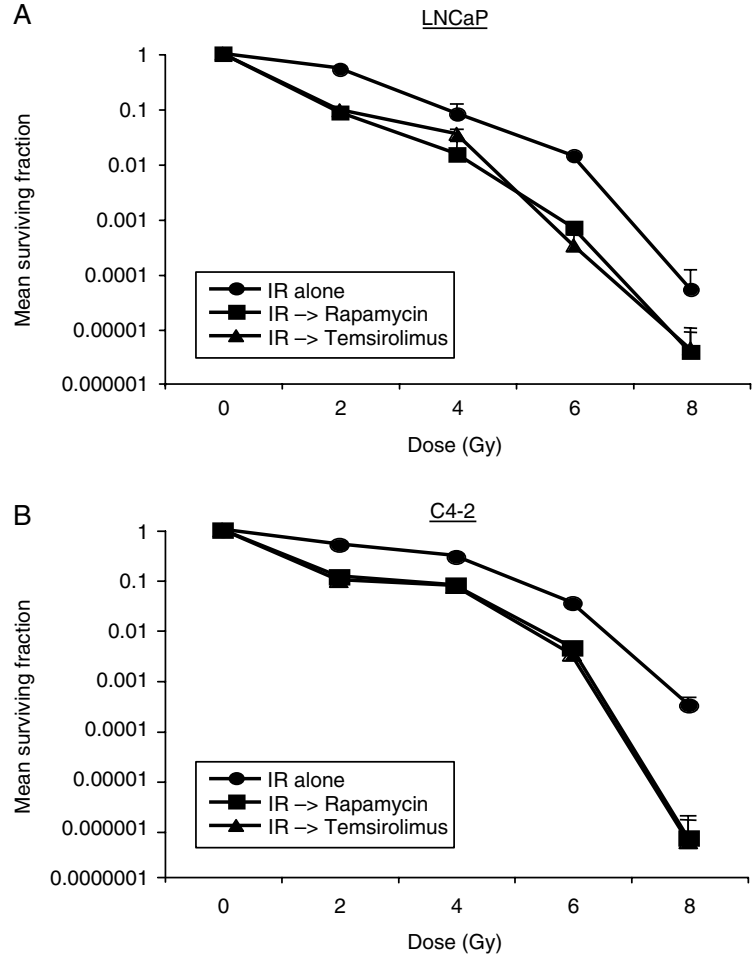

Figure 5 Clonogenic cell survival of both hormone therapysensitive and CRPC cells is reduced with mTOR inhibition following IR (A) LNCaP cells were serially diluted to appropriate concentrations and seeded in $50 \mathrm{ml}$ tissue culture flasks. $24 \mathrm{~h}$ later, cells were exposed to indicated doses of IR. After $24 \mathrm{~h}$, cells were treated with $10 \mathrm{nM}$ rapamycin, $10 \mathrm{nM}$ temsirolimus, or vehicle control. Cells were incubated for 14 days, fixed, stained with crystal violet, and colonies were counted. Colonies containing $>50$ cells were considered in the analysis. The data are represented on a semi-log scale, where the $x$-axis represents the dose of IR and the $y$-axis represents the mean surviving fraction and S.D. (B) C4-2 cells were seeded, treated, processed, counted, and analyzed as in (a).

in decreased replicative capacity of both HT-sensitive PCa and CRPC cells (Fig. 5). Only the schedule that proved to be most effective with respect to radiosensitization was utilized in the clonogenic assay. This was to ensure that any observed effects on sensitivity were not due to relative baseline plating efficiency to prior mTOR inhibitor administration. Together, these studies demonstrate that mTOR inhibition can radiosensitize PCa cells, and scheduling of the treatment alters the ultimate outcome as determined by both monitoring population doubling and clonogenic cell survival.

Despite the approved use of mTOR inhibitors for the treatment of renal clear cell carcinoma (RCC), there are few data regarding the impact of mTOR as a therapeutic target in PCa. However, a recent pharmacodynamic study (Armstrong et al. 2010) demonstrated that an mTOR inhibitor (Rapa) could be administered to men with localized PCa, attaining high intra-prostatic 
levels of the compound with minimal adverse effects and effectively limiting mTOR signaling as determined by S6 kinase phosphorylation, which is a downstream effector of mTOR activity involved in protein translation. While there was little significant biological effect in these tumors with regard to cellular outcomes (as determined by the assessment of proliferative and apoptotic indices), this may have been a result of the short course of treatment (14 days). Nonetheless, these clinical data demonstrate the feasibility of targeting mTOR in PCa cells, thus revealing a potentially fruitful platform for combination therapy. There are currently a number of clinical trials at various stages, some using mTOR inhibitors as single agents and others in combination with agents such as docetaxel or AR-directed strategies (as reviewed in Morgan et al. (2009)); however, none of these trials are investigating the combinatorial use of IR and mTOR inhibition in PCa. While this study indicates some modest impact of mTOR inhibitors as single agents, the most significant anti-tumor activity was observed in combination with IR. Therefore, the data presented herein demonstrating the radiosensitization of both HT-sensitive and CRPC cells in the clonogenic cell survival assay emphasize the importance of considering treatment schedule and provide the basis for clinical investigations. These data present a substantive advance, as there are no clinical agents currently approved, which confer sensitization to RT aside from androgen deprivation therapy (ADT). Of note, in CRPC cells cultured in conditions mimicking ADT, mTOR inhibition served as a radiosensitizer as well.

A critical finding herein was that the efficacy of mTOR inhibitors as a means to radiosensitize was significantly influenced by treatment schedule in both HT-sensitive PCa and CRPC cells. The evidence shown suggests that the observed schedule dependence can be attributed to the impact of mTOR inhibitors on cell cycle progression. The $G_{1}$ arrest induced by mTOR inhibitors prior to IR protected against radiation-induced cellular outcomes, whereas mTOR inhibitors in the adjuvant setting resulted in a more robust decrease in cell doubling. Interestingly, the effect of mTOR inhibitors was not influenced by PTEN status, as both PTEN-proficient (LAPC4) and PTEN-deficient (LNCaP) cells exhibited similar response to schedule-dependent combination therapy. Moreover, the impact of mTOR inhibition on radiosensitization was independent of p53 status as, in contrast to LNCaP cells, the LAPC4 model system lacks functional p53 (van Bokhoven et al. 2003). Schedule-dependent sensitization to DNA damaging therapies by mTOR inhibition is not without precedent.
It has been demonstrated that co-treatment of doxorubicin with an mTOR inhibitor was synergistic in T-cell lymphoma in vitro (Huang et al. 2010), as was adjuvant administration of mTOR inhibitor, compared to neo-adjuvant mTOR inhibition with these agents, which resulted in no synergistic effect on cellular outcomes. Additionally, it has been demonstrated that Tem administered to HT-sensitive PCa cells after docetaxel was more effective in limiting clonogenic cell survival, compared with concomitant treatment (Fung et al. 2009). These collective observations underscore the importance of assessing the impact of sequencing when combining mTOR inhibitors with genotoxic agents, especially with regard to the relative impact of these agents to alter cell cycle inhibition. As demonstrated herein, administration of mTOR inhibitors prior to radiation results in larger proportions of the cell populations being in relatively radioresistant portions of the cell cycle $\left(\mathrm{G}_{1}\right)$ and fewer cells in radiosensitive portions ( $S$ and $\mathrm{G}_{2} / \mathrm{M}$ ).

As demonstrated herein, clinically relevant doses of both Rapa and Tem exhibit single-agent cytostatic and cytotoxic effects in PCa cells and conferred schedule-dependent radiosensitization. The underlying mechanism(s) by which adjuvant administration of mTOR inhibition sensitizes cells to RT is the focus of ongoing investigation. Recently, it was demonstrated that mTOR is directly involved in the repair of DNA damage with respect to double-strand breaks, which occur frequently in cells exposed to IR (Chen et al. 2010), and these effects could therefore contribute to the radiosensitization observed in this study. Consonantly, it has been demonstrated that mTOR inhibition confers radiosensitization phenotypes in multiple tumor types (Ekshyyan et al. 2009, Nagata et al. 2010, Saunders et al. 2010), and that mTOR inhibition radiosensitizes soft tissue sarcoma and tumor vasculature (Murphy et al. 2009), which could have a similar impact on the response to RT. mTOR inhibitors also show cooperative effects with RTindependent DNA damaging agents, including doxorubicin (in T-cell lymphoma (Huang et al. 2010)), 5-fluorouracil and/or docetaxel (in gastric cancer (Matsuzaki et al. 2009)), carboplatin and paclitaxel (in head and neck cancer (Aissat et al. 2008)), and cisplatin (in hepatocellular carcinoma (Aissat et al. 2008)). In PCa cells, limited evidence suggests that mTOR inhibition can confer sensitization to doxorubicin (Grunwald et al. 2002), and combining mTOR inhibitors with docetaxel has been shown to be effective in limiting $\mathrm{PCa}$ cell growth in vitro and in vivo in a schedule-dependent manner (Fung et al. 2009). While mTOR inhibitors have been shown to 
cooperate with DNA damage in AR-negative PCa cells both in vitro and in vivo (Wu et al. 2005, Cao et al. 2006), the relevance of these models to the majority of human tumors, which retain AR, remains uncertain. One study has demonstrated that mTOR inhibition and docetaxel administration is an effective combination in an intra-tibial AR-positive model of PCa (Morgan et al. 2008), while the other has shown that combining mTOR inhibition and AR antagonistic therapy results in $\mathrm{PCa}$ cell apoptosis and delayed progression to castration resistance (Schayowitz et al. 2010). As such, mTOR inhibitors appear to harbor the capacity to improve responses to RT and selected DNA damageinducing therapeutics, as well as AR-directed strategies.

In summary, the studies presented herein demonstrate that mTOR inhibitors exhibit schedule-dependent effects on the RT response in PCa cells and confer significant radiosensitization effects when used in the adjuvant setting. Remarkably, the effects of mTOR inhibition as a means to achieve radiosensitization was conserved in both the HT-sensitive PCa and the CRPC settings, thus indicating that mTOR inhibitors may be an effective means to improve response to DNA damage-inducing therapeutic regimens in advanced disease. Combining these data herein provide the foundation for clinical investigation and illuminate new means by which PCa treatment may be improved.

\section{Supplementary data}

This is linked to the online version of the paper at http://dx. doi.org/10.1530/ERC-11-0072.

\section{Declaration of interest}

The authors declare that there is no conflict of interest that could be perceived as prejudicing the impartiality of the research reported.

\section{Funding}

This work was supported by NIH grants (CA099996 and CA116777 to K E K) and DOD Pre-doctoral Fellowships (PC094195 to M J S and PC094596 to M A A).

\section{Author contribution statement}

M J S, M A A, Y R L, A P D, and K E K conceived and designed the experiments. M J S, R D, D T H, and M A A performed the experiments. M J S, R D, D T H, Y R L, A P D, and $\mathrm{KE} \mathrm{K}$ analyzed the data. $\mathrm{KE} \mathrm{K}$ contributed reagents or analysis tools. M J S and K E K wrote the paper.

\section{Acknowledgements}

The authors thank the K Knudsen laboratory for critical input, especially R Schrecengost and J Goodwin, M Faradaugh for technical assistance, and the E Knudsen laboratory for commentary.

\section{References}

Aissat N, Le Tourneau C, Ghoul A, Serova M, Bieche I, Lokiec F, Raymond E \& Faivre S 2008 Antiproliferative effects of rapamycin as a single agent and in combination with carboplatin and paclitaxel in head and neck cancer cell lines. Cancer Chemotherapy and Pharmacology 62 305-313. (doi:10.1007/s00280-007-0609-2)

Allen GW, Howard AR, Jarrard DF \& Ritter MA 2007 Management of prostate cancer recurrences after radiation therapy-brachytherapy as a salvage option. Cancer 110 1405-1416. (doi:10.1002/cncr.22940)

Armstrong AJ, Netto GJ, Rudek MA, Halabi S, Wood DP, Creel PA, Mundy K, Davis SL, Wang T, Albadine R et al. 2010 A pharmacodynamic study of rapamycin in men with intermediate- to high-risk localized prostate cancer. Clinical Cancer Research 16 3057-3066. (doi:10.1158/ 1078-0432.CCR-10-0124)

Baldin V, Lukas J, Marcote MJ, Pagano M \& Draetta G 1993 Cyclin D1 is a nuclear protein required for cell cycle progression in G1. Genes and Development 7 812-821. (doi:10.1101/gad.7.5.812)

Beuvink I, Boulay A, Fumagalli S, Zilbermann F, Ruetz S, O'Reilly T, Natt F, Hall J, Lane HA \& Thomas G 2005 The mTOR inhibitor RAD001 sensitizes tumor cells to DNA-damaged induced apoptosis through inhibition of p21 translation. Cell 120 747-759. (doi:10.1016/j.cell. 2004.12.040)

van Bokhoven A, Varella-Garcia M, Korch C, Johannes WU, Smith EE, Miller HL, Nordeen SK, Miller GJ \& Lucia MS 2003 Molecular characterization of human prostate carcinoma cell lines. Prostate 57 205-225. (doi:10.1002/ pros.10290)

Bolla M, Collette L, Blank L, Warde P, Dubois JB, Mirimanoff RO, Storme G, Bernier J, Kuten A, Sternberg C et al. 2002 Long-term results with immediate androgen suppression and external irradiation in patients with locally advanced prostate cancer (an EORTC study): a phase III randomised trial. Lancet 360 103-106. (doi:10. 1016/S0140-6736(02)09408-4)

Braunstein S, Badura ML, Xi Q, Formenti SC \& Schneider RJ 2009 Regulation of protein synthesis by ionizing radiation. Molecular and Cellular Biology 29 5645-5656. (doi:10.1128/MCB.00711-09)

Cairns P, Okami K, Halachmi S, Halachmi N, Esteller M, Herman JG, Jen J, Isaacs WB, Bova GS \& Sidransky D 1997 Frequent inactivation of PTEN/MMAC1 in primary prostate cancer. Cancer Research 57 4997-5000.

Cao C, Subhawong T, Albert JM, Kim KW, Geng L, Sekhar KR, Gi YJ \& Lu B 2006 Inhibition of mammalian target of rapamycin or apoptotic pathway induces autophagy 
and radiosensitizes PTEN null prostate cancer cells. Cancer Research 66 10040-10047. (doi:10.1158/00085472.CAN-06-0802)

Chen Y, Sawyers CL \& Scher HI 2008 Targeting the androgen receptor pathway in prostate cancer. Current Opinion in Pharmacology 8 440-448. (doi:10.1016/j. coph.2008.07.005)

Chen H, Ma Z, Vanderwaal RP, Feng Z, Gonzalez-Suarez I, Wang S, Zhang J, Roti Roti JL \& Gonzalo S 2010 The mTOR inhibitor rapamycin suppresses DNA doublestrand break repair. Radiation Research 175 214-224.

Ekshyyan O, Rong Y, Rong X, Pattani KM, Abreo F, Caldito G, Chang JK, Ampil F, Glass J \& Nathan CO 2009 Comparison of radiosensitizing effects of the mammalian target of rapamycin inhibitor CCI-779 to cisplatin in experimental models of head and neck squamous cell carcinoma. Molecular Cancer Therapeutics 8 2255-2265. (doi:10.1158/1535-7163.MCT-08-1184)

Fung AS, Wu L \& Tannock IF 2009 Concurrent and sequential administration of chemotherapy and the mammalian target of rapamycin inhibitor temsirolimus in human cancer cells and xenografts. Clinical Cancer Research 15 5389-5395. (doi:10.1158/1078-0432.CCR08-3007)

Gao N, Zhang Z, Jiang BH \& Shi X 2003 Role of $\mathrm{PI} 3 \mathrm{~K} / \mathrm{AKT} / \mathrm{mTOR}$ signaling in the cell cycle progression of human prostate cancer. Biochemical and Biophysical Research Communications 310 1124-1132. (doi:10.1016/ j.bbrc.2003.09.132)

Grunwald V, DeGraffenried L, Russel D, Friedrichs WE, Ray RB \& Hidalgo M 2002 Inhibitors of mTOR reverse doxorubicin resistance conferred by PTEN status in prostate cancer cells. Cancer Research 62 6141-6145.

Hidalgo M \& Rowinsky EK 2000 The rapamycinsensitive signal transduction pathway as a target for cancer therapy. Oncogene 19 6680-6686. (doi:10.1038/ sj.onc.1204091)

Hirabayashi N, Noso Y, Niimi K, Nishiyama M, Yamaguchi M, Toge T, Niimoto M \& Hattori T 1987 Comparative studies on in vitro human tumor clonogenic assay (HTCA) and in vivo nude mouse-isotope assay (NM-IA). Japanese Journal of Surgery 17 104-109. (doi:10.1007/ BF02470649)

Huang JJ, Li ZM, Huang Y, Tian Y, He XX, Xiao J \& Lin TY 2010 Schedule-dependent inhibition of T-cell lymphoma cells by cotreatment with the mTOR inhibitor everolimus and anticancer drugs. Investigational New Drugs [in press]. (doi:10.1007/s10637-010-9558-4)

Jemal A, Siegel R, Xu J \& Ward E 2010 Cancer statistics. CA: A Cancer Journal for Clinicians 60 277-300. (doi:10. 3322/caac.20073)

Klein EA, Ciezki J, Kupelian PA \& Mahadevan A 2009 Outcomes for intermediate risk prostate cancer: are there advantages for surgery, external radiation, or brachytherapy? Urologic Oncology 27 67-71. (doi:10. 1016/j.urolonc.2008.04.001)
Klotz L 2006 Combined androgen blockade: an update. Urologic Clinics of North America 33 161-166. (doi:10. 1016/j.ucl.2005.12.001)

Knudsen KE \& Scher HI 2009 Starving the addiction: new opportunities for durable suppression of AR signaling in prostate cancer. Clinical Cancer Research 15 4792-4798. (doi:10.1158/1078-0432.CCR-08-2660)

Kremer CL, Klein RR, Mendelson J, Browne W, Samadzedeh LK, Vanpatten K, Highstrom L, Pestano GA \& Nagle RB 2006 Expression of mTOR signaling pathway markers in prostate cancer progression. Prostate 66 1203-1212. (doi:10.1002/pros.20410)

Kwok Y \& Yovino S 2010 Update on radiation-based therapies for prostate cancer. Current Opinion in Oncology 22 257-262. (doi:10.1097/CCO. 0b013e3283378c84)

Loblaw DA, Virgo KS, Nam R, Somerfield MR, Ben-Josef E, Mendelson DS, Middleton R, Sharp SA, Smith TJ, Talcott J et al. 2007 Initial hormonal management of androgensensitive metastatic, recurrent, or progressive prostate cancer: 2006 update of an American Society of Clinical Oncology Practice Guideline. Journal of Clinical Oncology 25 1596-1605. (doi:10.1200/JCO.2006.10.1949)

Matsuzaki T, Yashiro M, Kaizaki R, Yasuda K, Doi Y, Sawada T, Ohira M \& Hirakawa K 2009 Synergistic antiproliferative effect of mTOR inhibitors in combination with 5-fluorouracil in scirrhous gastric cancer. Cancer Science 100 2402-2410. (doi:10.1111/j.13497006.2009.01315.x)

McMenamin ME, Soung P, Perera S, Kaplan I, Loda M \& Sellers WR 1999 Loss of PTEN expression in paraffinembedded primary prostate cancer correlates with high Gleason score and advanced stage. Cancer Research 59 4291-4296.

Morgan TM, Pitts TE, Gross TS, Poliachik SL, Vessella RL \& Corey E 2008 RAD001 (everolimus) inhibits growth of prostate cancer in the bone and the inhibitory effects are increased by combination with docetaxel and zoledronic acid. Prostate 68 861-871. (doi:10.1002/pros.20752)

Morgan TM, Koreckij TD \& Corey E 2009 Targeted therapy for advanced prostate cancer: inhibition of the PI3K/Akt/ mTOR pathway. Current Cancer Drug Targets 9 237-249. (doi:10.2174/156800909787580999)

Motzer RJ, Escudier B, Oudard S, Hutson TE, Porta C, Bracarda S, Grunwald V, Thompson JA, Figlin RA, Hollaender $\mathrm{N}$ et al. 2008 Efficacy of everolimus in advanced renal cell carcinoma: a double-blind, randomised, placebo-controlled phase III trial. Lancet 372 449-456. (doi:10.1016/S0140-6736(08)61039-9)

Mousses S, Wagner U, Chen Y, Kim JW, Bubendorf L, Bittner M, Pretlow T, Elkahloun AG, Trepel JB \& Kallioniemi OP 2001 Failure of hormone therapy in prostate cancer involves systematic restoration of androgen responsive genes and activation of rapamycin sensitive signaling. Oncogene 20 6718-6723. (doi:10. 1038/sj.onc.1204889) 
Murphy JD, Spalding AC, Somnay YR, Markwart S, Ray ME \& Hamstra DA 2009 Inhibition of mTOR radiosensitizes soft tissue sarcoma and tumor vasculature. Clinical Cancer Research 15 589-596. (doi:10.1158/1078-0432. CCR-08-1019)

Nagata Y, Takahashi A, Ohnishi K, Ota I, Ohnishi T, Tojo T \& Taniguchi S 2010 Effect of rapamycin, an mTOR inhibitor, on radiation sensitivity of lung cancer cells having different p53 gene status. International Journal of Oncology 37 1001-1010. (doi:10.3892/ijo_00000751)

van der Poel HG, Hanrahan C, Zhong H \& Simons JW 2003 Rapamycin induces Smad activity in prostate cancer cell lines. Urological Research 30 380-386. (doi:10.1007/ s00240-002-0282-1)

Pourmand G, Ziaee AA, Abedi AR, Mehrsai A, Alavi HA, Ahmadi A \& Saadati HR 2007 Role of PTEN gene in progression of prostate cancer. Urology Journal 4 95-100.

Qian DZ, Rademacher BL, Pittsenbarger J, Huang CY, Myrthue A, Higano CS, Garzotto M, Nelson PS \& Beer TM 2010 CCL2 is induced by chemotherapy and protects prostate cancer cells from docetaxel-induced cytotoxicity. Prostate 70 433-442. (doi:10.1002/pros.21077)

Sansal I \& Sellers WR 2004 The biology and clinical relevance of the PTEN tumor suppressor pathway. Journal of Clinical Oncology 22 2954-2963. (doi:10. 1200/JCO.2004.02.141)

Saunders PO, Cisterne A, Weiss J, Bradstock KF \& Bendall LJ 2011 The mTOR inhibitor RAD001 (everolimus) synergizes with chemotherapeutic agents, ionizing radiation and proteasome inhibitors in pre-B ALL. Haematologica 96 69-77.

Schayowitz A, Sabnis G, Goloubeva O, Njar VC \& Brodie AM 2010 Prolonging hormone sensitivity in prostate cancer xenografts through dual inhibition of AR and mTOR. British Journal of Cancer 103 1001-1007. (doi:10.1038/sj.bjc.6605882)

Sharma A, Yeow WS, Ertel A, Coleman I, Clegg N, Thangavel C, Morrissey C, Zhang X, Comstock CE, Witkiewicz AK et al. 2010 The retinoblastoma tumor suppressor controls androgen signaling and human prostate cancer progression. Journal of Clinical Investigation 120 4478-4492. (doi:10.1172/JCI44239)

Shen C, Lancaster CS, Shi B, Guo H, Thimmaiah P \& Bjornsti MA 2007 TOR signaling is a determinant of cell survival in response to DNA damage. Molecular and Cellular Biology 27 7007-7017. (doi:10.1128/MCB.00290-07)

Sinclair WK \& Morton RA 1966 X-ray sensitivity during the cell generation cycle of cultured Chinese hamster cells. Radiation Research 29 450-474. (doi:10.2307/3572025)

Sircar K, Yoshimoto M, Monzon FA, Koumakpayi IH, Katz RL, Khanna A, Alvarez K, Chen G, Darnel AD, Aprikian AG et al. 2009 PTEN genomic deletion is associated with
p-Akt and AR signalling in poorer outcome, hormone refractory prostate cancer. Journal of Pathology 218 505-513. (doi:10.1002/path.2559)

Taplin ME 2007 Drug insight: role of the androgen receptor in the development and progression of prostate cancer. Nature Clinical Practice. Oncology 4 236-244. (doi:10. 1038/ncponc0765)

Tirado OM, Mateo-Lozano S, Sanders S, Dettin LE \& Notario V 2003 The PCPH oncoprotein antagonizes the proapoptotic role of the mammalian target of rapamycin in the response of normal fibroblasts to ionizing radiation. Cancer Research 63 6290-6298.

Whang YE, Wu X, Suzuki H, Reiter RE, Tran C, Vessella RL, Said JW, Isaacs WB \& Sawyers CL 1998 Inactivation of the tumor suppressor PTEN/MMAC1 in advanced human prostate cancer through loss of expression. PNAS 95 5246-5250. (doi:10.1073/pnas.95.9.5246)

Wilson AP, Ford CH, Newman CE \& Howell A 1984 A comparison of three assays used for the in vitro chemosensitivity testing of human tumours. British Journal of Cancer 49 57-63. (doi:10.1038/bjc.1984.9)

Wu L, Birle DC \& Tannock IF 2005 Effects of the mammalian target of rapamycin inhibitor CCI-779 used alone or with chemotherapy on human prostate cancer cells and xenografts. Cancer Research 65 2825-2831. (doi:10.1158/0008-5472.CAN-04-3137)

Xu W, Ngo L, Perez G, Dokmanovic M \& Marks PA $2006 a$ Intrinsic apoptotic and thioredoxin pathways in human prostate cancer cell response to histone deacetylase inhibitor. PNAS 103 15540-15545. (doi:10.1073/pnas. 0607518103)

Xu Y, Chen SY, Ross KN \& Balk SP $2006 b$ Androgens induce prostate cancer cell proliferation through mammalian target of rapamycin activation and post-transcriptional increases in cyclin D proteins. Cancer Research 66 7783-7792. (doi:10.1158/0008-5472.CAN-05-4472)

Yau TM, Kim SC, Nygaard OF, Gregg EC \& Crissman HA 1980 Correlation of cell cycle parameters with radiation sensitivity in a series of murine L5178Y cells.

International Journal of Radiation Biology and Related Studies in Physics, Chemistry, and Medicine 37 429-435. (doi:10.1080/09553008014550501)

Yuan X \& Balk SP 2009 Mechanisms mediating androgen receptor reactivation after castration. Urologic Oncology 27 36-41. (doi:10.1016/j.urolonc.2008.03.021)

Yung WK 1989 In vitro chemosensitivity testing and its clinical application in human gliomas. Neurosurgical Review 12 197-203. (doi:10.1007/BF01743984)

\author{
Received in final form 22 August 2011 \\ Accepted 8 September 2011 \\ Made available online as an Accepted Preprint \\ 8 September 2011
}

